We are now ready to derive a formula $N(c)$. Let $q_{i}$ be any odd prime for which -7 is a quadratic residue, and $n_{j}$ any for which -7 is a quadratic non-residue. Let $c$, any positive odd integer, be written in the form

$$
c=7^{0} \prod_{i, j} q_{i}^{e_{i}} n_{j}^{f_{j}}, \quad g \geqq 0, i \geqq 0, j \geqq 0, e_{i} \geqq 1, f_{j} \geqq 1 .
$$

Theorem 2. Let $N(1)=13, N(3)=8$, and for $c$ any odd integer greater than 3 , let $N(c)$ be the least common multiple of all the factors $7^{\circ}$, $q_{i}-1, n_{j}+1, q_{i}^{e_{i}-1}, n_{j}^{f_{j}-1}$, then if $n>N(c),\left|a_{n}\right| \neq c$.

By [1], $\left|a_{n}\right|>1$ if $n>13$. By Theorem 1 , if $n>8\left|a_{n}\right| \neq 3$. By Lemmas $12,13,14$, and $15, a_{N(c)} \equiv 0(\bmod c)$. Suppose $\left|a_{n}\right|=c, c>3$. By Theorem 1, this is true for only one $n$. By Lemma 7 , this $n$ must be a factor of $N(c)$, therefore $n \leqq N(c)$. Thus for all values of $c$, if $n>N(c)$, then $\left|a_{n}\right| \neq c$.

\title{
REFERENCES
}

1. Th. Skolem, P. Chowla, and D. J. Lewis, The Diophantine equation $2^{n-2}-7=x^{2}$ and related problems, Proc. Amer. Math. Soc. 10 (1959), 663-669.

2. P. Chowla, $A$ class of Diophantine equations, Proc. Nat. Acad. Sci. U.S.A. 45 (1959), 569-570.

UNIVERSITY OF HAWAII

\section{TWO NEW REPRESENTATIONS OF THE PARTITION FUNCTION}

\section{BASIL GORDON}

MacMahon [1] defined a two-rowed partition of the positive integer $n$ as a representation of the form $n=\sum_{i=1}^{r} a_{i}+\sum_{j=1}^{s} b_{j}$ where the $a_{i}$ and $b_{j}$ are positive integers subject to the conditions $r \geqq s, a_{i} \geqq a_{i+1}$, $b_{j} \geqq b_{j+1}, a_{i} \geqq b_{i}$. Such partitions may be conveniently visualized by placing the summands on two rows, the $a_{i}$ on the top row and the $b_{j}$ on the bottom row, with each $b_{i}$ immediately beneath $a_{i}$. Thus for $n=3$ the partitions in question are (omitting + signs)

$$
\begin{aligned}
& 3,21,2,111,11 . \\
& 11
\end{aligned}
$$

In this note the following two theorems will be proved.

THEOREM 1. The number of two-rowed partitions of $n$ satisfying $a_{i}>a_{i+1}, b_{j}>b_{j+1}$ is $p(n)$, the ordinary partition function of $n$.

Received by the editors January 6, 1962 . 
THEOREM 2. The number of two-rowed partitions of $n$ with $a_{i}, b_{j}$ odd and also satisfying $a_{i}>a_{i+1}, b_{j}>b_{j+1}$ is $p([n / 2])$.

As an example of Theorem 1, take $n=5$, getting the partitions

$$
\begin{array}{ccccc}
5,41, & 4, & 32, & 3, & 31, \\
1 & 2 & 1 & 2
\end{array}
$$

There are $p(5)=7$ of these. Again if $n=9$ the partitions of Theorem 2 are

$$
\begin{aligned}
& \text { 9, 71, 531, 53, 51, } \\
& 1 \quad 1,3
\end{aligned}
$$

and there are $p(4)=5$ of these.

Proof of Theorem 1. Two-rowed partitions of $n$ with $a_{i}>a_{i+1}$, $b_{i}>b_{i+1}$ will be referred to henceforth as $\delta$-partitions. If the summands of such a partition are all written on one line we obtain an ordinary partition of $n$ in which no integer appears more than twice as a part.

Conversely, let $\pi$ be an ordinary partition of $n$ in which no integer appears more than twice as a part. Such a partition is of the form $\pi: n=c_{1}+\cdots+c_{k}+2 c_{k+1}+\cdots+2 c_{l}$, where $c_{1}>c_{2}>\cdots>c_{k}, c_{k+1}$ $>c_{k+2}>\cdots>c_{l}$, and where $c_{i} \neq c_{j}$ for $i \neq j$. Then the $c_{i}$ can be distributed on 2 rows to form a $\delta$-partition, perhaps in several ways. Since the parts $c_{k+1}, \cdots, c_{l}$ must clearly appear once on each row, the number of $\delta$-partitions arising from $\pi$ depends only on $k$. We will now show that it equals ${ }_{k} C_{[k / 2]}$, the maximum binomial coefficient of order $k$. For this purpose it is convenient to introduce a more general quantity $Q(k, m)$, the number of $\delta$-partitions formed from $c_{1}, \cdots, c_{k}$ with the number of parts on the top row exceeding that of parts on the bottom row by at least $m$. We thus wish to show that $Q(k, 0)$ $={ }_{k} C_{[k / 2]}$. Now for $m>0, Q(k, m)$ satisfies the recurrence

$$
Q(k, m)=Q(k-1, m-1)+Q(k-1, m+1) .
$$

This can be seen by observing that if $c_{k}$ is on the top row, the remaining $c$ 's can be distributed in $Q(k-1, m-1)$ ways, and if it is on the bottom, in $Q(k-1, m+1)$ ways. When $m=0$ the recurrence still holds if we make the convention that $Q(k,-1)=Q(k, 0)$. This recurrence enables us to determine $Q(k, m)$ from the initial conditions $Q(1, m)=1$ when $m \leqq 1, Q(1, m)=0$ when $m \geqq 2$. The general formula is $Q(k, m)$ $={ }_{k} C_{[(k-m) / 2]}$, which gives the desired result when $m=0$.

Next observe that when $k=2 s+1$ is odd, ${ }_{k} C_{[k / 2]}={ }_{2 s+1} C_{s}$ is the number of ways in which the parts $c_{1}, \cdots, c_{k}$ can be separated into two 
sets $A, B$ of cardinalities $s$ and $s+1$ respectively. If $k=2 s$ is even, then ${ }_{k} C_{[k / 2]}={ }_{28} C_{8}$ is the number of ways of separating $c_{1}, \cdots, c_{k}$ into two sets $A, B$ of cardinality $s$ (with the separation $B, A$ being regarded as distinct from $A, B)$. Consider now the full partition $\pi: n=c_{1}+\cdots$ $+c_{k}+2 c_{k+1}+\cdots+2 c_{k+l}$, with $k+2 l$ parts. Every separation of $c_{1}, \cdots, c_{k}$ into two sets $A, B$ can be uniquely extended to a separation of all the parts into sets $A^{\prime}, B^{\prime}$ of distinct integers (by placing one $c_{j}$ in $A$ and the other in $B$ for each $\left.j=k+1, \cdots, k+l\right)$. Hence the number $\Delta(n)$ of $\delta$-partitions of $n$ is the same as the number of partitions of the form

$n=d_{1}+\cdots+d_{h}+e_{1}+\cdots+e_{h+1}\left(d_{1}>\cdots>d_{h}, e_{1}>\cdots>e_{h+1}\right)$

or of the form

$$
d_{1}+\cdots+d_{h}+e_{1}+\cdots+e_{h}\left(d_{1}>\cdots>d_{h}, e_{1}>\cdots>e_{h}\right)
$$

(here $d_{1}+\cdots+d_{h}+e_{1}+\cdots+e_{h}$ and $e_{1}+\cdots+e_{h}+d_{1}+\cdots+d_{h}$ are counted as distinct). It follows that if $F_{h}(\alpha)$ denotes the number of partitions of $\alpha$ into exactly $h$ distinct parts, then the number $\Delta(n)$ of $\delta$-partitions of $n$ has the value

$$
\Delta(n)=\sum_{h=0}^{\infty} \sum_{\alpha=0}^{n}\left\{F_{h}(\alpha) F_{h}(n-\alpha)+F_{h}(\alpha) F_{h+1}(n-\alpha)\right\} .
$$

The generating function of $F_{h}(\alpha)$ is

$$
\sum_{\alpha=0}^{\infty} F_{h}(\alpha) x^{\alpha}=\frac{x^{\left(h^{2}+h\right) / 2}}{(1-x) \cdots\left(1-x^{h}\right)}(|x|<1)
$$

(cf. [2, Theorem 346]), and therefore

$$
\begin{aligned}
& \sum_{n=0}^{\infty} \Delta(n) x^{n} \\
& =\sum_{h=0}^{\infty}\left\{\frac{x^{h^{2}+h}}{(1-x)^{2} \cdots\left(1-x^{h}\right)^{2}}+\frac{x^{(h+1)^{2}}}{(1-x)^{2} \cdots\left(1-x^{h}\right)^{2}\left(1-x^{h+1}\right)}\right\} \\
& =\sum_{r=0}^{\infty}\left\{\frac{x^{r^{2}+r}}{(1-x)^{2} \cdots\left(1-x^{r}\right)^{2}}+\frac{x^{r^{2}}\left(1-x^{r}\right)}{(1-x)^{2} \cdots\left(1-x^{r-1}\right)^{2}\left(1-x^{r}\right)^{2}}\right\} \\
& =\sum_{h=0}^{\infty} \frac{x^{h^{2}}}{(1-x)^{2} \cdots\left(1-x^{h}\right)^{2}} \cdot
\end{aligned}
$$

By virtue of an identity of Durfee (cf. [2, Theorem 351]), the lat- 
ter series is equal to $\sum_{n=0}^{\infty} p(n) x^{n}$. The identity theorem for power series now yields $\Delta(n)=p(n)$, and the proof is complete.

I am indebted to Professor T. S. Motzkin and Mr. C. Sudler for suggesting simplifications in my original proof of Theorem 1.

Proof of Theorem 2. Letting $\Delta_{0}(n)$ denote the number of $\delta$ partitions of $n$ into odd parts, we have to show that

$$
\sum_{n=0}^{\infty} \Delta_{0}(n) x^{n}=(1+x) f\left(x^{2}\right)
$$

where $f(x)=\sum_{n=0}^{\infty} p(n) x^{n}$; indeed it then follows at once that $\Delta_{0}(n)$ $=p([n / 2])$.

It is evident that the proof of Theorem 1 can be repeated, with the only difference being that all the parts $c_{1}, \cdots, c_{l}$ are required to be odd. We thus obtain the expression

$$
\Delta_{0}(n)=\left\{\sum_{h=0}^{\infty} \sum_{\alpha=0}^{n} G_{h}(\alpha) G_{h}(n-\alpha)+G_{h}(\alpha) G_{h+1}(n-\alpha)\right\},
$$

where $G_{h}(\alpha)$ is the number of partitions of $\alpha$ into exactly $h$ distinct odd parts. The generating function of $G_{h}(\alpha)$ is

$$
\sum_{\alpha=0}^{\infty} G_{h}(\alpha) x^{\alpha}=\frac{x^{h^{2}}}{\left(1-x^{2}\right)\left(1-x^{4}\right) \cdots\left(1-x^{2 h}\right)}
$$

(cf. [2, p. 279]). Hence

$$
\begin{aligned}
& \sum_{n=0}^{\infty} \Delta_{0}(n) x^{n} \\
& =\sum_{h=0}^{\infty}\left\{\frac{x^{2 h^{2}}}{\left(1-x^{2}\right)^{2} \cdots\left(1-x^{2 h}\right)^{2}}+\frac{x^{h^{2}+(h+1)^{2}}}{\left(1-x^{2}\right)^{2} \cdots\left(1-x^{2 h}\right)^{2}\left(1-x^{2 h+2}\right)}\right\} \\
& =\sum_{h=0}^{\infty} \frac{x^{2 h^{2}}}{\left(1-x^{2}\right)^{2} \cdots\left(1-x^{2 h}\right)^{2}} \\
& \quad+x \sum_{h=0}^{\infty} \frac{x^{2 h(h+1)}}{\left(1-x^{2}\right)^{2} \cdots\left(1-x^{2 h}\right)^{2}\left(1-x^{2 h+2}\right)} .
\end{aligned}
$$

The first series is $f\left(x^{2}\right)$ by Durfee's identity. To show that the second term is $x f\left(x^{2}\right)$ we must prove that

$$
\sum_{h=0}^{\infty} \frac{x^{h(h+1)}}{(1-x)^{2} \cdots\left(1-x^{h}\right)^{2}\left(1-x^{h+1}\right)}=f(x) .
$$


This can be seen combinatorially in much the same way as Durfee's identity. If $\pi$ is a partition of $n$, its Ferrars graph contains a maximal $h \times(h+1)$ rectangle $R$, and the remaining points of the graph constitute two tails. If the tail to the right of $R$ has $\alpha$ points and the tail below $R$ has $\beta$ points, there are $P_{h}(\alpha) P_{h+1}(\beta)$ ways of adjoining the tails to $R$, where $P_{k}(m)$ is the number of partitions of $m$ into at most $k$ parts. Hence

$$
p(n)=\sum_{h=0}^{\infty} \sum_{\alpha+\beta=n-h(h+1)} F_{h}(\alpha) F_{h+1}(\beta),
$$

and passing to the generating functions we obtain (1). This completes the proof of Theorem 2.

Concluding REMARKs. Chaundy [3] has dealt with the more general problem of determining the number of $r$-rowed partitions of $n$ with parts decreasing by at least $\rho$ along rows and at least $\kappa$ along columns. Theorem 1 is the case $r=2, \rho=1, \kappa=0$. I am unable to see that Chaundy's argument is correct, owing to the failure of his expression (11) to satisfy the proper initial conditions. In any event, Theorem 1 falls under the case $\rho+\kappa=1$, in which Chaundy's formula becomes indeterminate.

Theorem 2 can easily be transformed into the statement that the number of plane partitions of $n$ possessing $x y$-symmetry, and whose parts do not exceed 2 , is $p([n / 2])$. In this form it appears as a "theorem" in [1, p. 269]. However, the "proof" rests on the results of $\$ 520$ which, as the reader is warned in $\$ 521$, have not yet been rigorously established.

Finally, Theorems 1 and 2 suggest the problem of finding directly a 1:1 correspondence between the $\delta$-partitions and ordinary partitions of $n$, and between the odd $\delta$-partitions of $n$ and the ordinary partitions of $[n / 2]$. As in the case of the Rogers-Ramanujan identities, this has not yet been done, and appears to be difficult.

\section{REFERENCES}

1. P. A. MacMahon, Combinatory analysis, Cambridge Univ. Press, New York, 1916.

2. G. H. Hardy and E. M. Wright, An introduction to the theory of numbers, 4th ed., Oxford Univ. Press, Oxford, 1960.

3. T. W. Chaundy, Partition-generating functions, Quart. J. Math. 2 (1931), 234240 .

University of California, Los ANGeles 